\title{
Shock-Induced Electromotive Force in Aqueous Solution: from Weak to Concentrated Solutions
}

\author{
T. Ogura, Y. Hironaka, M. Nicol, and K. Kondo \\ Materials and Structures Laboratory, Tokyo Institute of Technology, Nagatsuta, Midori, Yokohama 226, Japan
}

\begin{abstract}
Shock-induced electromotive forces were measured in water and potassium-fluoride aqueous solutions. While we obtain the same result of former researches for pure water, the magnitude of the emf signal tended to increase with concentration in weak aqueous solutions. These phenomena may be explained with the assumption that emf is initiated by the change in Fermi level of measuring electrode under high pressure polarizing the adjacent liquid. The emf signals generated in concentrated solutions behaved unlike those of dilute solutions. Some models are proposed as the source of shock induced emf signals.

[electromotive force, dilute solution, concentrated solution, dielectric constant, Fermi energy]
\end{abstract}

\section{Introduction}

Shock-induced electromotive forces (EMF's) have been studied by many workers and in many solids or liquids. EMF signals generated by the passage of shock waves are complicated and dramatically different in different materials. Several sources of EMF's have been suggested, for instance, dielectric polarization, piezoelectricity, thermoelectromotive force of the measuring electrodes and other phenomena. The origins, however, are not understood and may not be simple.

We took water and aqueous solutions of electrolytes as samples for shock wave experiments. Water is interesting because of its large molecular dipole moment and strong hydrogen bonds which determine its three dimensional-structure. Harris and Presles [1] have previously indicated that the origin of shock induced emf signal of water was the dielectric polarization which was caused by the rotation of molecules. In contrast with this, Breusov et al. [2] reported that the intensity of electrical signal varied with the kind of material of the measuring electrodes and concluded the origin was the phenomenon concerning the electrodes. We felt that, if ordering dipole moment causes the shock induced EMF's, the large dipole moment of water molecule might generate especially large EMF's. For EMF's at the boundary between a sample material and measuring electrode, it also is important to know the electrical conductivity of the material. From this point of view, we also wanted to observe aqueous solutions to study how changing the conductivity affected the shock induced EMF's.

\section{Experimental Procedure}

a) Sample holder, The sample holder consists of driver electrode, measuring electrode (10 mm-dia), and guard ring which reduces the influence of edge effect of the measuring electrode. All of them were made of copper and finished to mirror-like surfaces by using the alumina polishing paper. Spacers separated the electrodes were made of polycarbonate. The distance between the driver and measuring electrode was 1 $\mathrm{mm}$. The driver plate was grounded and connected to the measuring electrode through a $50 \Omega$ electrical resistor. The difference of EMF generated across this resistor by the passage of the shock wave was measured by the low impedance $(50 \Omega$ ) digital oscilloscope. The guard ring was connected to the ground through $25 \Omega$.

b) Samples, The sample water (Wako Chemical) contained impurities no more than $4.2 \mathrm{ppm}$ of electrolytes. For the aqueous solutions, we chose potassium-fluoride (Wako Chemical), we did not use potassium-chloride to prevent the copper electrodes from spoiling.

c) Experiment, Shock waves were generated with a singlestage powder gun and copper or tungsten impactors. The pressure dependence of the EMF was measured from 5 to 10 $\mathrm{GPa}$ for pure water and the concentration dependence was measured at the same shock pressure $(7 \mathrm{GPa})$ for solutions with mole fraction of $\mathrm{KF}, X_{K F}$, up to $2.0 \times 10^{-2}$.

\section{Results}

Pure water, The pressure dependence of the peak EMF is shown in Fig.1. The magnitude of EMF's is almost consistent with the results of former researchers [1],[2]. In analysis of the data we used the model by Allison [3], which assumes the dielectric polarization on the shock wave front, $\mathrm{P}_{S}{ }^{0}$. From his model we could calculate $\mathrm{P}_{\mathrm{S}}{ }^{0}$, the relaxation time of polarization, $\tau$, and dielectric constant of the sample in shocked state, $\varepsilon^{\prime}$.

Figure 2 is the plot of $P_{S}{ }^{0}$ with pressure. We can see the linear relation of $\mathrm{P}_{S}{ }^{0}$ with pressure. $\tau$ was of the order of sub-micro seconds, and $\varepsilon^{\prime}$ of water was extremely large, around 300 . We could not identify an obvious relationship between $\tau$ and $\varepsilon^{\prime}$ (Table.1).

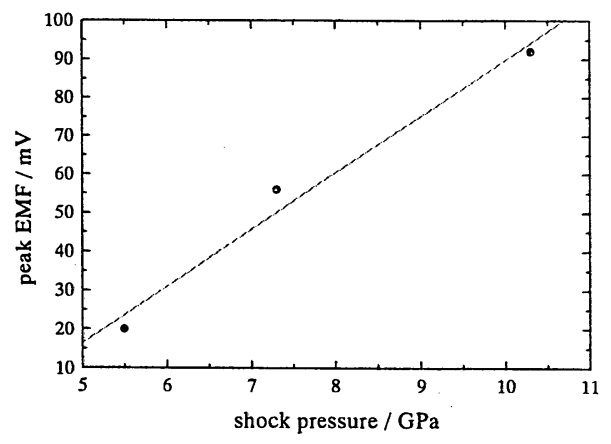

Fig.1. The pressure dependence of peak EMF generated in pure water in the range of 5 to $10 \mathrm{GPa}$ for $1-\mathrm{mm}$ thick samples. 


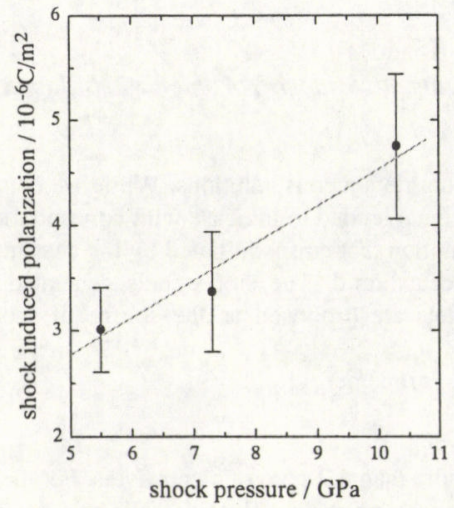

Fig.2. The pressure dependence of shock induced polarization, $\mathrm{P}_{\mathrm{S}}^{0}$, generated in pure water at the shock wave fronts. Error bars are acquired from fitting conditions to theoretical curves.

\begin{tabular}{cccc}
\hline Pressure / GPa & $\tau / \mu \mathrm{s}$ & $\varepsilon^{\prime}$ & $\mathrm{P}_{\mathrm{S}}{ }^{0} / 10^{-6} \mathrm{C} / \mathrm{m}^{2}$ \\
\hline 5.5 & 0.05 & 280 & 3.0 \\
7.3 & 0.08 & 320 & 3.4 \\
10.3 & 0.14 & 240 & 4.8 \\
\hline
\end{tabular}

Table.1. The parameters of shocked water calculated from Allison's model.

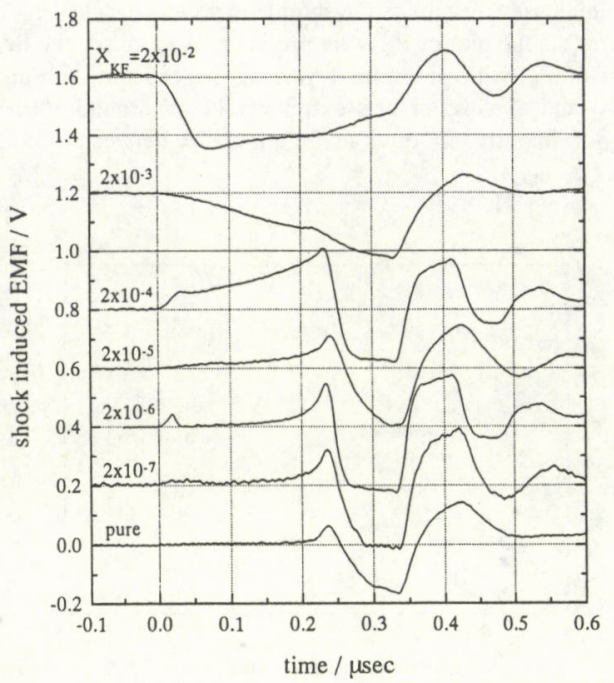

Fig.3. The concentration dependence of EMF generated in aqueous $\mathrm{KF}$ solution at $7 \mathrm{GPa}$. The samples were 1 -mm thick.
Aqueous solutions, Figure 3 shows the raw data acquired for several concentrations of $\mathrm{KF}$ aqueous solutions shocked to 7 $\mathrm{GPa}$. Time zero corresponds to the time when shock wave entered the liquid. The peaks of the signals around $0.24 \mu \mathrm{s}$ indicate when shock wave reached the end of the sample and entered the measuring electrode. For dilute solutions $\left(X_{K F}\right.$ below $2 \times 10^{-4}$ ), the magnitude of EMF increased with the concentration of the solution. However the polarity of the EMF changed and was negatively going in concentrated range $\left(\mathrm{X}_{\mathrm{KF}}>2 \times 10^{-3}\right)$.

Water is ionized and has large electrical conductivity under high pressure. The result of Hamann and Linton [4] was adopted in our analysis, and we used values of the dielectric constant of water from Kirkwood's theory [5]. The effect of electrolyte on dielectric constant is negligible in our experimental region [6]. Figure 4 depicts the raw data and the curves from the models by Allison [3] and Breusov et al [2]. According to the model by

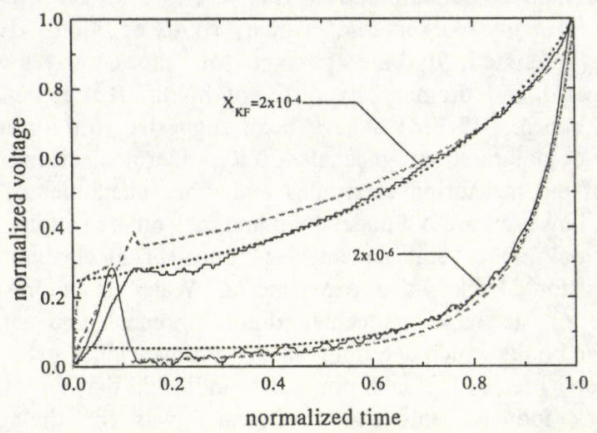

Fig.4. Fitting the theoretical curves to the experimental data for $X_{K F}=2 \times 10^{-6}$ and $2 \times 10^{-4}$ (- :experiment; $\cdots$ :Allison; $-\cdots$ Breusov $\left(\varepsilon^{\prime}=100\right.$ was used as dielectric constant of shocked water)). Axes are normalized.

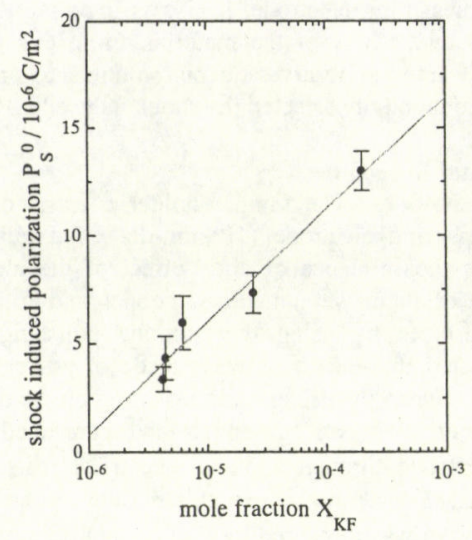

Fig.5. The $X_{K F}-\mathrm{P}_{\mathrm{S}}{ }^{0}$ relation for dilute $\mathrm{KF}$ solution at $7 \mathrm{GPa}$ (up to $X_{K F}=1.0 \times 10^{-3}$ ). 
Breusov et al. the source of the EMF is the two electrodes, and we can calculate the electrical potential difference between electrodes. The average value obtained for these four experiments was $270 \pm 30 \mathrm{mV}$ (Table.2). The concentration dependence of $\mathrm{P}_{\mathrm{s}}{ }^{0}$ calculated from Allison's model is in Fig.5.

\begin{tabular}{cc}
\hline $\begin{array}{c}\text { Mole fraction } \\
\mathrm{X}_{\mathrm{KF}}\end{array}$ & $\begin{array}{c}\text { potential difference } \\
/ \mathrm{mV}\end{array}$ \\
$2.0 \times 10^{-7}$ & 240 \\
$2.0 \times 10^{-6}$ & 290 \\
$2.0 \times 10^{-5}$ & 250 \\
$2.0 \times 10^{-4}$ & 290 \\
\hline
\end{tabular}

Table.2. The potential difference between shocked and unshocked electrodes calculated from Breusov's model at $7 \mathrm{GPa}$ (up to $\mathrm{X}_{\mathrm{KF}}=2.0 \times 10^{-4}$ ). The value of dielectric constant of shocked water, $\varepsilon^{\prime}$, is 100 .

\section{Discussion}

The curves from both models fit the data relatively well (Fig.4). The dielectric constant of shocked water is calculated to be around 100 at $7 \mathrm{GPa}$ from Kirkwood's theory [5]. When we admit the dielectric polarization as the source of EMF's, dielectric constant of shocked water was computed to be about 300 from Allison's model. An explanation of this extremely large value may be the failure of this model to consider the electrical conductivity of the sample. By considering it the calculated dielectric constant will decrease. The calculated relaxation time of the polarization (sub-micro second) is quite longer than the characteristic time of water. We can acquire the linearity of the logarithm of $X_{K F}-\mathrm{P}_{\mathrm{S}}^{0}$ relation in dilute concentrations. This may have some relation with any change in property of water, for example viscosity, owing to the electrolyte.

If we adopt that the EMF's arise from the two electrodes, the source of EMF should be due to the change of property of electrode under high pressure and temperature. Former researchers have studied the pressure dependence of band structure of copper. Davis et al. [7] calculated that Fermi energy increased by $0.24 \mathrm{eV}$ when lattice spacing decreased by $1 \%$. Zallen [8] reported that the d-band - Fermi level separation increased by the rate less than $1 \times 10^{-6} \mathrm{eV} / \mathrm{bar}$ and temperature dependence was quite small. From their reports we can estimate the change of Fermi level between shocked and unshocked copper electrodes to be almost $1.5 \mathrm{eV}$ or $0.070 \mathrm{eV}$ at $7 \mathrm{GPa}$ respectively (these values are large or small relative to our data, electrical potential difference between electrodes is $270 \pm 30 \mathrm{mV}$ ), so we can not determine whether the EMF is due to the change of Fermi level of copper or not. But the effect of this change on the electrode should be real.

For the more concentrated solutions $\left(\mathrm{X}_{\mathrm{KF}}>2.0 \times 10^{-3}\right)$, the polarity of the signal changed. In every other system studied in this laboratory [9], the polarization signals are positive. We are not sure why the EMF's became negative, but it may be the influence of the electrical double layer that consists of $\mathrm{F}^{-}$anion and $\mathrm{K}^{+}$cation on the copper electrodes.

\section{Acknowledgment}

We are grateful to Japan Science and Technology Corporation that has supported this research.

\section{References}

[1] P. Harris and H. Presles, J. Chem. Phys., 77, 5157 (1982).

[2] O. Breusov, A. Dremin, V. Kochnev, S. Nabatov, and V. Yakushev, Sov. Phys -JETP., 34, 591 (1972).

[3] F. Allison, J. Appl. Phys., 36, 2111 (1968).

[4] S. Hamann and M. Linton, Trans. Faraday Soc., 65, 2186 (1969).

[5] D. Eisenberg and W. Kauzmann, The Structure and Properties of Water, Oxford University Press, Oxford, 1969.

[6] F. Franks, Water A Comprehensive Treatise : volume 3 , Aqueous Solutions of Simple Electrolytes, Plenum Press, New York - London, 1973.

[7] H. Davis, J. Faulkner, and H. Joy, Phys. Rev., 167, 601 (1968).

[8] R. Zallen, Opt. Prop. Electron. Struct. Met. Alloys, Proc. Intl. Colloq, 164 (1966).

[9] K. Saxena, Y. Hironaka, H. Hirai, and K.Kondo, Appl. Phys. Lett, 68, 920 (1996). 